\title{
Interpreting a covid-19 test result
}

\author{
Jessica Watson GP and National Institute for Health Research doctoral research fellow ${ }^{1}$, Penny F \\ Whiting associate professor in clinical epidemiology ${ }^{1}$, John E Brush professor of internal medicine ${ }^{2}$
}

${ }^{1}$ Centre for Academic Primary Care, Bristol Medical School, University of Bristol, Bristol, UK; ${ }^{2}$ Sentara Healthcare and Eastern Virginia Medical School, Norfolk, VA, USA

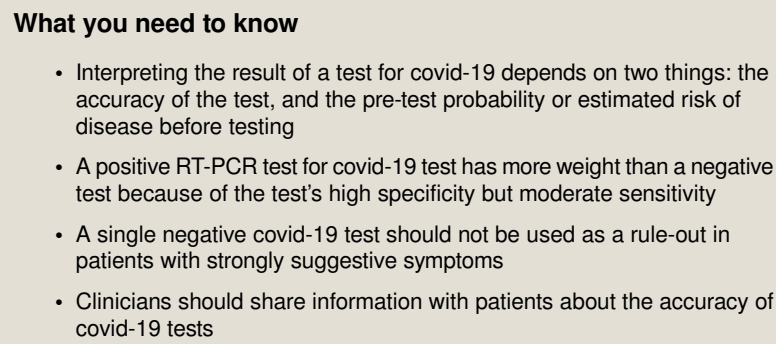

Across the world there is a clamour for covid-19 testing, with Tedros Adhanom Ghebreyesus, director general of the World Health Organization, encouraging countries to "test, test, test." The availability of the complete genome of covid-19 early in the epidemic facilitated development of tests to detect viral RNA. ${ }^{2}$ Multiple assays with different gene targets have been developed using reverse transcriptase polymerase chain reaction (RT-PCR). ${ }^{3}$ These viral RNA tests use samples usually obtained from the respiratory tract by nasopharyngeal swab, to detect current infections. Serology blood tests to detect antibodies indicating past infection are being developed; these will not be considered in depth in this article.

Testing for covid-19 enables infected individuals to be identified and isolated to reduce spread, ${ }^{4}$ allows contact tracing for exposed individuals, ${ }^{5}$ and provides knowledge of regional and national rates of infection to inform public health interventions. However, questions remain on how to apply test results to make optimal decisions about individual patients.

\section{Search strategy}

This article was produced at speed to address an urgent need to address uncertainties in testing for covid-19. We searched Pubmed using the terms "covid", "SARS-CoV-2", "sensitivity", "specificity", "diagnosis", "test", and "PCR", and KSR evidence using terms for covid and test. This was supplemented by discussion with colleagues undertaking formal systematic reviews into covid-19 diagnosis.

\section{How accurate are test results?}

No test gives a $100 \%$ accurate result; tests need to be evaluated to determine their sensitivity and specificity, ideally by comparison with a "gold standard." The lack of such a clear-cut "gold-standard" for covid-19 testing makes evaluation of test accuracy challenging.

A systematic review of the accuracy of covid-19 tests reported false negative rates of between $2 \%$ and $29 \%$ (equating to sensitivity of 71-98\%), based on negative RT-PCR tests which were positive on repeat testing. ${ }^{6}$ The use of repeat RT-PCR testing as gold standard is likely to underestimate the true rate of false negatives, as not all patients in the included studies received repeat testing and those with clinically diagnosed covid-19 were not considered as actually having covid- $19 .{ }^{6}$ Accuracy of viral RNA swabs in clinical practice varies depending on the site and quality of sampling. In one study, sensitivity of RT-PCR in 205 patients varied, at $93 \%$ for broncho-alveolar lavage, $72 \%$ for sputum, $63 \%$ for nasal swabs, and only $32 \%$ for throat swabs. ${ }^{7}$ Accuracy is also likely to vary depending on stage of disease ${ }^{8}$ and degree of viral multiplication or clearance. ${ }^{9}$ Higher sensitivities are reported depending on which gene targets are used, and whether multiple gene tests are used in combination. ${ }^{310}$ Reported accuracies are much higher for in vitro studies, which measure performance of primers using coronavirus cell culture in carefully controlled conditions. ${ }^{2}$

The lack of a clear-cut "gold-standard" is a challenge for evaluating covid-19 tests; pragmatically, clinical adjudication may be the best available "gold standard," based on repeat swabs, history, and contact with patients known to have covid-19, chest radiographs, and computed tomography scans. Inevitably this introduces some incorporation bias, where the test being evaluated forms part of the reference standard, and this would tend to inflate the measured sensitivity of these tests. ${ }^{11}$ Disease prevalence can also affect estimates of accuracy: tests developed and evaluated in populations with high prevalence (eg, secondary care) may have lower sensitivity when applied in a lower prevalence setting (eg, primary care). ${ }^{11}$ 
One community based study of 4653 close contacts of patients with covid-19 tested RT-PCR throat swabs every 48 hours during a 14 day quarantine period. Of 129 eventually diagnosed with covid-19 by RT-PCR, 92 (71.3\%) had a positive test on the first throat swab, equating to a sensitivity of $71 \%$ in this lower prevalence, community setting. ${ }^{12}$

Further evidence and independent validation of covid-19 tests are needed. ${ }^{13}$ As current studies show marked variation and are likely to overestimate sensitivity, we will use the lower end of current estimates from systematic reviews, ${ }^{6}$ with the approximate numbers of $70 \%$ for sensitivity and $95 \%$ for specificity for illustrative purposes.

\section{What do clinicians need to know to understand a test result?}

Sensitivity and specificity can be confusing terms that may be misunderstood ${ }^{14}$ (see supplementary file 'Definitions and formulae for calculating measures of test accuracy'). Sensitivity is the proportion of patients with disease who have a positive test, or the true positive rate. Specificity is the proportion of patients without disease who have a negative test, or true negative rate. These terms describe the operating characteristics of a test and can be used to gauge the credibility of a test result. They can be combined to calculate likelihood ratios, which are dimensionless numbers that indicate the strength of a positive or negative test result. ${ }^{15}$ For calculating probabilities, a likelihood ratio can be used as a multiplier to convert pre-test odds to post-test odds. Positive likelihood ratios greater than 1 are progressively stronger, with 10 representing a very strong positive test result. Negative likelihood ratios less than 1 are also progressively stronger, with 0.1 representing a very strong negative test result. In the case of the nasopharyngeal swab RNA test for covid-19, the positive likelihood ratio is about 14, which is excellent. ${ }^{6}$ A positive covid-19 test result should be very compelling. The negative likelihood ratio is 0.3 , which is a moderate result, but not nearly as compelling as a positive result because of the moderate sensitivity (about 70\%) of the covid-19 test.

Interpretation of a test result depends not only on the characteristics of the test itself but also on the pre-test probability of disease. Clinicians use a heuristic (a learned mental short cut) called anchoring and adjusting to settle on a pre-test probability (called the anchor). They then adjust this probability based on additional information. This heuristic is a useful short cut but comes with the potential for bias. When people fail to estimate the pre-test probability and only respond to a piece of new information, they commit a fallacy called base-rate neglect. Another fallacy called anchoring is failing adequately to adjust one's probability estimate, given the strength of new information. Likelihood ratios can give a clinician an idea of how much to adjust their probability estimates. Clinicians intuitively use anchoring and adjusting thoughtfully to estimate pre- and post-test probabilities unconsciously in everyday clinical practice. However, faced with a new and unfamiliar disease such as covid-19, mental short cuts can be uncertain and unreliable and public narrative about the definitive nature of testing can skew perceptions.

Figure 1 shows how a clinician's thinking about a patient's probability should shift, based on either a positive or negative test result for covid-19. First, the clinician should estimate a pre-test probability, using knowledge of local rates of covid-19 infection from nationa ${ }^{16}$ and regional ${ }^{17}$ data and patients' symptoms and signs, ${ }^{18}$ likelihood of alternative diagnoses, and history of exposure to covid-19. After choosing a pre-test probability on the $\mathrm{x}$ axis, one should then trace up to either the upper curve for a positive test result or the lower curve for a negative test result, then trace over to the y axis to read the estimate for post-test probability. The figure shows that the shift in the probability is asymmetric, with a positive test result having a greater impact than a negative test result, owing to the modest sensitivity and negative likelihood ratio of the RNA test.

The infographic (fig 2) shows the outcomes when 100 people with a pre-test probability of $80 \%$ are tested for covid- 19 using natural frequencies, which are generally easier to understand. Online calculators are available which allow clinicians to adjust pre-test probability, sensitivity, and specificity to estimate post-test probability ${ }^{19}$

\section{What else should clinicians consider when interpreting test results?}

\section{A single negative test result may not be informative if the pre-test probability is high}

A 52 year old general practitioner in London develops a cough, intermittent fever, and malaise. On day 2 of his illness he receives a nasopharyngeal swab test for covid-19, which is reported as negative. His cough and low-grade fever persist but he feels systemically well enough to return to work. What should he do?

Pre-test probability is high in someone with typical symptoms of covid-19, an occupational risk of exposure, and working in a high prevalence region, and negative test results can therefore be misleading. Table 1 shows that for a pre-test probability of $90 \%$, someone with a negative test has a $74 \%$ chance of having covid-19; with two negative tests this risk is still around $47 \%$. If this doctor were to return to work and subsequently the test was confirmed as a false negative, then the decision to work would potentially have significant consequences for his patients, colleagues, and everyone with whom he came into contact. It is therefore safest for this GP with strongly suggestive symptoms to self-isolate in line with guidelines for covid-19, even though his test results are negative. This case illustrates the fallacy of base-rate neglect; it can be tempting to trust the results of an "objective" test more than one's own "subjective" clinical judgement. In general, during this pandemic, pre-test probabilities of covid-19 will be high, particularly in high prevalence secondary care settings.

\section{A possible alternative diagnosis will reduce the pre-test probability}

A 73 year old woman with severe chronic obstructive pulmonary disease (COPD) and a chronic cough develops acute shortness of breath and slight worsening of her non-productive cough. She reports no fever, has no known exposure to covid-19, and no recent travel. She presents to an emergency department where she is acutely short of breath. A chest radiograph shows possible infiltrates in the right upper and middle lung fields. She is admitted and placed in isolation on droplet precautions. She requires intubation for worsening respiratory distress. Initial nasopharyngeal covid-19 testing is negative. Should she remain in isolation on droplet precautions?

This patient has an alternative possible diagnosis: community-acquired pneumonia. Given her lack of other risk factors or clinical symptoms, and chest radiography findings we therefore estimate her pre-test probability at about $50 \%$. One negative test reduces this risk to $24 \%$, the patient therefore has an additional independently sampled nasopharyngeal swab RNA 
test which was negative, giving a post-test probability after two negative tests of less than $10 \%$. She is treated with antibiotics and continues to recover.

\section{What are the implications for practice and policy?}

While positive tests for covid-19 are clinically useful, negative tests need to be interpreted with caution, taking into account the pre-test probability of disease. This has important implications for clinicians interpreting tests and policymakers designing diagnostic algorithms for covid-19. The Chinese handbook of covid-19 prevention and treatment states "if the nucleic acid test is negative at the beginning, samples should continue to be collected and tested on subsequent days. ${ }^{20}$ False negatives carry substantial risks; patients may be moved into non-covid-19 wards leading to spread of hospital acquired covid-19 infection, ${ }^{21}$ carers could spread infection to vulnerable dependents, and healthcare workers risk spreading covid-19 to multiple vulnerable individuals. Clear evidence-based guidelines on repeat testing are needed, to reduce the risk of false negatives.

Clinicians should ensure that patients are counselled about the limitations of tests (box 1). Patients with a single negative test but strongly suggestive symptoms of covid-19 should be advised to self-isolate in keeping with guidelines for suspected covid- 19 .

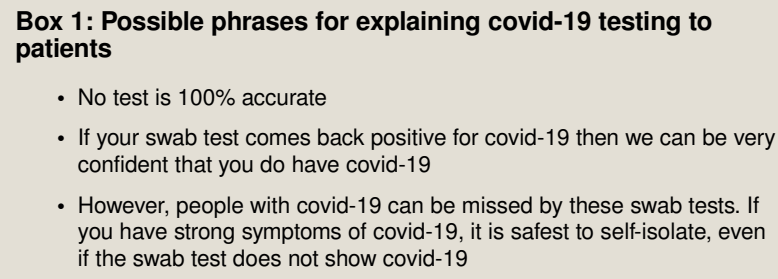

\section{What is the role of serology tests?}

Serology tests, which detect immunoglobulins including IgG and IgM, are under development, ${ }^{22-24}$ with the aim of detecting individuals who have had previous infection and therefore theoretically developed immunity. The time course and accuracy of serology tests are still under investigation, but the same principles of incorporating the test result with the clinical impression applies. False positive serology tests could cause false reassurance, behaviour change, and disease spread. If suitable accuracy can be established, the benefits of these antibody tests include establishing when healthcare workers are immune, helping to inform decisions about the lifting of lockdowns, and allowing the population to return to work. ${ }^{25}$

The WHO message "test, test, test" is important from a population perspective; low sensitivity can be accounted for when assessing burden of disease. However RT-PCR tests have limitations when used to guide decision making for individual patients. Positive tests can be useful to "rule-in" covid-19, a negative swab test cannot be considered definitive for "ruling out."

\section{How patients were involved in the creation of this article}

Patients with covid-19 or possible covid-19 were not involved in the writing of this paper for practical reasons

\section{Education into practice}

What is the protocol for covid-19 testing in your organisation? How do you explain covid-19 test results to patients?

Reflect on a recent clinical case of suspected covid-19-what was your estimated pre-test probability? How did this alter with the results of tests?

Author contributorship: JW JB and PW contributed to the conception of the work, $\mathrm{JW}$ ran the searches and wrote the first draft of the paper with assistance from JB. PW developed the tools for fig 2. JB, JW, and PW all contributed to the revised drafts of the paper and approved the final version for submission.

Acknowledgments: The authors would like to acknowledge Jon Deeks for helpful discussions at an early point in writing this article and Richard Lehman for suggestions and comments on a draft of this article.

Competing interests The $B M J$ has judged that there are no disqualifying financial ties to commercial companies. The authors declare the following other interests: JB has given Grand Rounds talks on medical reasoning and has published a book The Science of the Art of Medicine: A Guide to Medical Reasoning for which he receives royalties. JW has no competing interests to declare.

Further details of The BMJ policy on financial interests are here: https://www.bmj. com/about-bmj/resources-authors/forms-policies-and-checklists/declarationcompeting-interests

Funding: JW is funded by a doctoral research fellowship from the National Institute for Health Research. The views expressed in this publication are those of the authors and not necessarily those of the NHS, the National Institute for Health Research, Health Education England, or the Department of Health.

Patient consent: The cases in this article are fictitious and therefore no consent was needed.

Provenance and peer review: Commissioned, based on an idea from the author; externally peer reviewed.

BBC News. WHO head: 'Our key message is: test, test, test'. 2020. https://www.bbc.co. uk/news/av/world-51916707/who-head-our-key-message-is-test-test-test

2 Corman VM, Landt O, Kaiser M, etal Detection of 2019 novel coronavirus (2019-nCoV) by real-time RT-PCR. Eur Surveill 2020;25:2000045.

3 Vogels $\mathrm{CBF}$, Brito AF, Wyllie AL, et al. Analytical sensitivity and efficiency comparisons of SARS-COV-2 qRT-PCR assays. medRxiv 20048108. 2020 10.1101/2020.03.30.20048108\%J

4 Day M. Covid-19: identifying and isolating asymptomatic people helped eliminate virus in Italian village. BMJ 2020;368:m1165. 10.1136/bmj.m1165 32205334

5 Pollock AM, Roderick P, Cheng KK, Pankhania B. Covid-19: why is the UK government ignoring WHO's advice?BMJ 2020;368:m1284. 10.1136/bmj.m1284 32229543

6 Arevalo-Rodriguez I, Buitrago-Garcia D, Simancas-Racines D, et al. False-negative results of initial RT-PCR assays for covid-19: a systematic review. medRxiv 20066787. 2020 10.1101/2020.04.16.20066787\%J

7 Wang W, Xu Y, Gao R, etal . Detection of SARS-CoV-2 in different types of clinical specimens[JAMA.]. JAMA 2020. 10.1001/jama.2020.3786. 32159775

8 Sethuraman N, Sundararaj Stanleyraj J, Ryo A. Interpreting diagnostic tests for SARS-CoV-2. JAMA 202010.1001/jama.2020.8259.

9 Wölfel R, Corman VM, Guggemos W, etal . Virological assessment of hospitalized patients with COVID-2019. Nature 2020. 10.1038/s41586-020-2196-x. 32235945

10 Chan JF, Yip CC, To KK, etal . Improved molecular diagnosis of COVID-19 by the novel, highly sensitive and specific COVID-19-RdRp/Hel real-time reverse

transcription-polymerase chain reaction assay validated in vitro and with clinical specimens. J Clin Microbiol 2020. 10.1128/JCM.00310-20. 32132196

11 Usher-Smith JA, Sharp SJ, Griffin SJ. The spectrum effect in tests for risk prediction, screening, and diagnosis. BMJ 2016;353:i3139. 10.1136/bmj.i3139 27334281

12 Luo L, Liu D, Liao X-I, et al. Modes of contact and risk of transmission in COVID-19 among close contacts. medRxiv 20042606. 2020. 10.1101/2020.03.24.20042606\%J

13 FIND. SARS-CoV-2 diagnostics: performance data 2020. 2020. https://www.finddx.org/ covid-19/dx-data

14 Casscells W, Schoenberger A, Graboys TB. Interpretation by physicians of clinical laboratory results. N Engl J Med 1978;299:999-1001. 10.1056/NEJM197811022991808 692627

15 Brush J. The Science of the Art of Medicine: A Guide to Medical Reasoning Manakin-Sabot. Dementi Milestone Publishing, Inc, 2015.

16 Worldometer. Covid-19 Coronavirus pandemic data. 2020. https://www.worldometers. info/coronavirus/\#countries)

17 Public Health England. COVID-19: track coronavirus cases. 2020. https://www.gov.uk/ government/publications/covid-19-track-coronavirus-cases

18 Centre for Evidence-Based Medicine. COVID-19 signs and symptoms tracker. 2020 https://www.cebm.net/covid-19/covid-19-signs-and-symptoms-tracker/

19 Calculator MT. 2020. https://calculator.testingwisely.com/playground

20 First Affiliated Hospital of Zhejiang University School of Medicine. Handbook of COVID-19 Prevention and Treatment. 2020. https://gmcc.alibabadoctor.com/prevention-manual

21 Nacoti M, Ciocca A, Giupponi A, et al. At the epicenter of the covid-19 pandemic and humanitarian crises in Italy: changing perspectives on preparation and mitigation. 2020; 10.1056/CAT.20.0080 
22 Guo L, Ren L, Yang S, etal . Profiling early humoral response to diagnose novel coronavirus disease (COVID-19). Clin Infect Dis 2020;ciaa310. 10.1093/cid/ciaa310. 32198501

23 Zhao J, Yuan Q, Wang H, etal . Antibody responses to SARS-CoV-2 in patients of novel coronavirus disease 2019. Clin Infect Dis 2020;ciaa344. 10.1093/cid/ciaa344. 32221519

$24 \mathrm{Li} Z$, Yi Y, Luo X, etal . Development and clinical application of a rapid IgM-IgG combined antibody test for SARS-CoV-2 infection diagnosis. J Med Virol 2020.

10.1002/jmv.25727. 32104917
25 Petherick A. Developing antibody tests for SARS-CoV-2. Lancet 2020;395:1101-2. 10.1016/S0140-6736(20)30788-1 32247384

Published by the BMJ Publishing Group Limited. For permission to use (where not already granted under a licence) please go to http://group.bmj.com/group/rights-licensing/ permissions 


\section{Table}

Table 1| Pre- and post- test probabilities for covid-19 RT-PCR tests, calculations based on a sensitivity of $70 \%$ and specificity of $95 \%$ Pre-test probability Post-test probability, negative test Post-test probability, two independently negative tests Post-test probability positive test

\begin{tabular}{lccc}
5 & 1.6 & 0.5 & 42 \\
\hline 15 & 5 & 2 & 71 \\
\hline 25 & 10 & 3 & 82 \\
\hline 50 & 24 & 9 & 93 \\
\hline 75 & 49 & 23 & 98 \\
\hline 90 & 74 & 47 & 99 \\
\hline
\end{tabular}




\section{Figures}

\section{----- Positive test \\ - - Negative test}

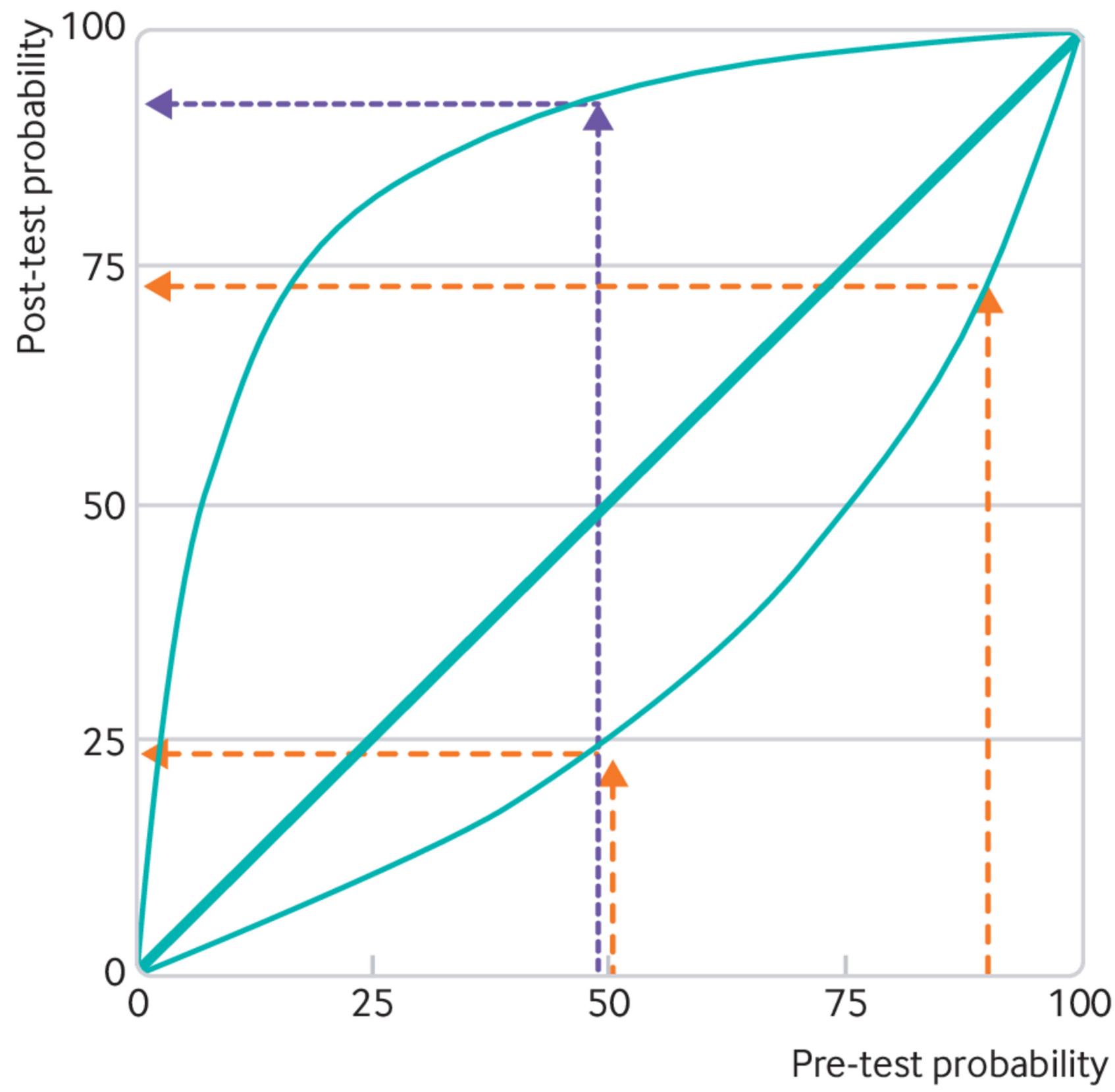

Fig 1 Leaf plot for covid-19 RT-PCR tests based on a sensitivity of $70 \%$ and specificity of $95 \%$. The $x$ axis gives the estimated pre-test probability of covid-19 based on the clinical details. The post-test probability is obtained by tracing up and across to the $y$ axis from the lower curve for a negative test, or to the upper curve for a positive test result. The dashed lines illustrate pre-test probability of $90 \%$ (clinical case 1 ) and $50 \%$ (clinical case 2 ) 
100 people at risk of covid-19

Pre-test probability $80 \%$

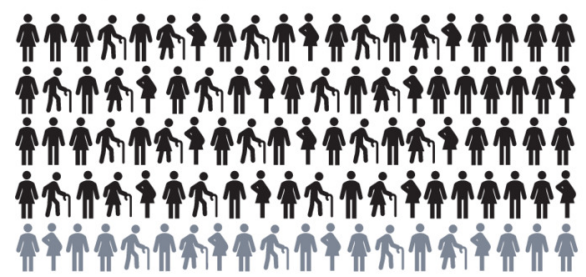

if 80 people have covid-19

if 20 people do not have covid-19

They are tested for covid-19 using the RT-PCR test:

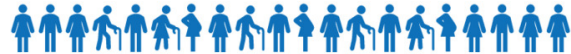

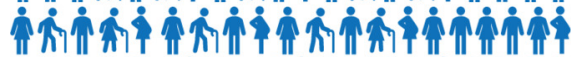

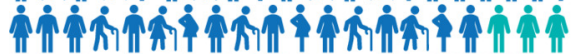

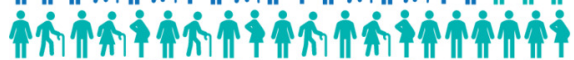

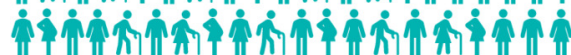

i 57 people have a test result suggesting that they have covid-19 (test positive)

in 43 people have a test result suggesting that they do not have covid-19 (test negative)

But who actually has covid-19?

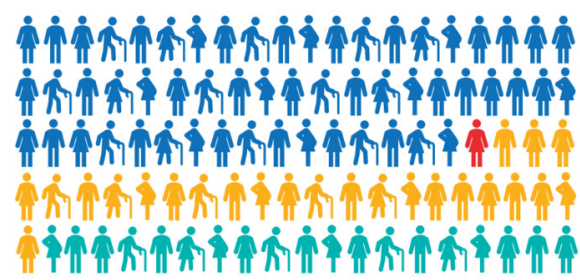

\begin{tabular}{|c|c|}
\hline $\begin{array}{l}\text { Diagnosis } \\
56 \text { people who test } \\
\text { positive have covid-19 } \\
\text { ("true positive") }\end{array}$ & $\begin{array}{l}\text { Consequences } \\
\text { Appropriately told to } \\
\text { self-isolate }\end{array}$ \\
\hline $\begin{array}{l}1 \text { person who tests positive } \\
\text { does not have covid-19 } \\
\text { ("false positive") }\end{array}$ & $\begin{array}{l}\text { Told they need to self-isolate } \\
\text { when they would be safe to } \\
\text { go out }\end{array}$ \\
\hline $\begin{array}{l}24 \text { people who test } \\
\text { negative have covid-19 } \\
\text { ("false negative") }\end{array}$ & $\begin{array}{l}\text { Told they do not need to } \\
\text { self-isolate and so go out and } \\
\text { infect more people }\end{array}$ \\
\hline $\begin{array}{l}19 \text { people who test negative } \\
\text { do not have covid-19 } \\
\text { ("true negative") }\end{array}$ & $\begin{array}{l}\text { Told they do not need to } \\
\text { self-isolate and are safe to go } \\
\text { out without infecting more } \\
\text { people }\end{array}$ \\
\hline
\end{tabular}

Fig 2 Infographic showing outcomes of 100 people who are tested for covid-19 